\title{
McLaren's Improved Snub Cube and Other New Spherical Designs in Three Dimensions
}

\author{
R. H. Hardin and N. J. A. Sloane \\ Mathematical Sciences Research Center, AT\&T Bell Laboratories, \\ Murray Hill, NJ 07974, USA \\ rhh/njas@research.att.com
}

\begin{abstract}
Evidence is presented to suggest that, in three dimensions, spherical 6-designs with $N$ points exist for $N=24,26, \geq 28 ; 7$-designs for $N=24,30,32,34, \geq 36 ; 8$-designs for $N=36,40,42$, $\geq 44 ; 9$-designs for $N=48,50,52, \geq 54 ; 10$-designs for $N=60,62$, $\geq 64 ; 11$-designs for $N=70,72, \geq 74$; and 12-designs for $N=84, \geq 86$. The existence of some of these designs is established analytically, while others are given by very accurate numerical coordinates. The 24-point 7-design was first found by McLaren in 1963, andalthough not identified as such by McLaren-consists of the vertices of an "improved" snub cube, obtained from Archimedes' regular snub cube (which is only a 3-design) by slightly shrinking each square face and expanding each triangular face. 5-designs with 23 and 25 points are presented which, taken together with earlier work of Reznick, show that 5-designs exist for $N=12,16,18,20, \geq 22$. It is conjectured, albeit with decreasing confidence for $t \geq 9$, that these lists of $t$-designs are complete and that no others exist. One of the constructions gives a sequence of putative spherical $t$-designs with $N=12 \mathrm{~m}$ points $(m \geq 2)$ where $N=\frac{1}{2} t^{2}(1+o(1))$ as $t \rightarrow \infty$.
\end{abstract}

\section{Introduction}

A set of $N$ points $\wp=\left\{P_{1}, \ldots, P_{N}\right\}$ on the unit sphere

$$
\Omega_{d}=S^{d-1}=\left\{x=\left(x_{1}, \ldots, x_{d}\right) \in \mathbb{R}^{d}: x \cdot x=1\right\}
$$

forms a spherical $t$-design if the identity

$$
\int_{\Omega_{d}} f(x) d \mu(x)=\frac{1}{N} \sum_{i=1}^{N} f\left(P_{i}\right)
$$

(where $\mu$ is a uniform measure on $\Omega_{d}$ normalized to have total measure 1) holds for all 
polynomials $f$ of degree $\leq t$ [10], [13], [6], Section 3.2. In this paper we are concerned only with the case $d=3$. $^{1}$

It is trivial that 1-designs exist if and only if $N \geq 2$, and Mimura [23] showed that 2-designs exist if and only if $N=4, \geq 6$. Bajnok [2] found 3-designs for $N=6,8$, $\geq 10$ and conjectured that they do not exist for $N=7$ and $9 .^{2}$ That 3-designs do not exist for $N \leq 5$ is a consequence of the lower bounds

$$
\begin{aligned}
& N \geq \frac{(t+1)(t+3)}{4} \quad \text { if } t \text { odd, } \\
& N \geq \frac{(t+2)^{2}}{4} \quad \text { if } t \text { even, }
\end{aligned}
$$

if $t \neq 1,2,3,5$ the right-hand sides of (2a), (2b) can be increased by 1 ,

which were established in [10], [3], and [4]. In [16] we showed that 4-designs exist for $N=12,14, \geq 16$, and conjectured that no others exist. Reznick [25] showed that 5-designs exist for $N=12,16,18,20,22,24, \geq 26$. We have found 5-designs with $N=23$ and 25 (see Table 1), and, our search having repeatedly failed in the remaining cases, conjecture that 5-designs do not exist for $N=13-15,17,19$, and 21. Bajnok [1] gave a general construction for $t$-designs on $\Omega_{3}$, but his designs (described in Section 5) are much larger than ours.

Following Reznick [25], we make use of the fact that a set of points $\left\{P_{i}\right\}$ forms a spherical $t$-design if and only if the polynomial identities

$$
\frac{1}{N} \sum_{i=1}^{N}\left(P_{i} \cdot x\right)^{2 s}=\left(\prod_{j=0}^{s-1} \frac{2 j+1}{2 j+3}\right)(x \cdot x)^{s}
$$

and

$$
\frac{1}{N} \sum_{i=1}^{N}\left(P_{i} \cdot x\right)^{2 \bar{s}+1}=0
$$

hold, where $s$ and $\bar{s}$ are defined by $\{2 s, 2 \bar{s}+1\}=\{t-1, t\}$ (see [13] and p. 114 of [24]).

\section{Summary of Results}

Let $\tau(N)$ denote the largest value of $t$ for which an $N$-point three-dimensional spherical $t$-design exists. Since a $t$-design is also a $t^{\prime}$-design for all $t^{\prime} \leq t$, an $N$-point spherical $t$-design exists if and only if $\tau(N) \geq t$.

Our main results are summarized in Table 1 , which gives what we believe are the values of $\tau(N)$ for $N \leq 100$. The assertions made in the first sentence of the abstract can then be simply read off the table. The table also gives, in columns 4 and 5 , the largest

\footnotetext{
${ }^{1}$ We are in the process of producing an analogous table of four-dimensional designs; these will be described elsewhere.

${ }^{2}$ In his talk Bajnok actually claimed to have a 9-point 3-design, but he now believes that this was a mistake.
} 
symmetry group we have found for such a design (using the notation of [8]), and in some cases a list of the sizes of the orbits under this group and a description of the polyhedron formed by the points. In most cases the designs found were not unique.

For every value of $N$ in the table we have found very accurate numerical coordinates for a putative spherical $t$-design with $t$ equal to the value given in column 2. Furthermore, after a considerable amount of searching, we have been unable to find a $(t+1)$-design, and so we conjecture that the entries in column 2 do indeed give the exact values of $\tau(N)$.

In a number of cases we have proved that there is a spherical $t$-design that is very close to our numerical approximation. To do this we reduce (3a), (3b) to a set of simultaneous algebraic equations, and then show either algebraically (with the help of Maple [5]) or by interval methods (using Intbis [20]) that these equations do have a solution in the neighborhood of the approximate solution. Examples will be found in the next two sections.

A symbol V1 in the third column of Table 1 indicates that we have an algebraic proof of the existence of the design, V2 that we have a proof by interval methods, and V3 that we have a numerical solution with discrepancy $\Delta(\wp)$ (defined below) at most $10^{-26}$. References to the literature indicate who first proved the existence of some spherical $t$-design with this number of points (not necessarily the particular design described in the table).

The numerical coordinates for these $t$-designs were found by a modified version of the Hooke and Jeeves [19] "pattern search" optimizer that we have already used to search for spherical codes [18] and experimental designs [15]-[17]. Let $\mathcal{F}_{d}(0 \leq d \leq t)$ denote the set of $\left(\begin{array}{c}d+2 \\ 2\end{array}\right)$ monomials $f=x_{1}^{e_{1}} x_{2}^{e_{2}} x_{3}^{e_{3}}$ of degree $d$, and let $\Delta_{f}(\wp)$ be the difference between the right and left sides of (1) for this $f$ for a set of points $\wp=\left\{P_{1}, \ldots, P_{N}\right\}$. The criterion we used was to minimize

$$
\sum_{f \in \mathcal{F}_{t-1}} \frac{(t-1) !}{e_{1} ! e_{2} ! e_{3} !} \Delta_{f}(\wp)^{2}+\sum_{f \in \mathcal{F}_{t}} \frac{t !}{e_{1} ! e_{2} ! e_{3} !} \Delta_{f}(\wp)^{2},
$$

since $\wp$ is a spherical $t$-design if and only if this sum vanishes. (The multinomial coefficients make the sums rotationally invariant.) As a check we also computed the discrepancy of the points,

$$
\Delta(\wp)=\sum_{d=1}^{t} \sum_{f \in \mathcal{F}_{1}} \Delta_{f}(\wp)^{2} .
$$

In practice we have found that, in the range of Table 1 , if $\Delta(\wp)<10^{-16}$, then there is a spherical $t$-design $\wp^{*}$ very close to $\wp$.

Incidentally, it would be nice to have a formal version of this: a theorem giving an explicit bound $\Delta_{0}(t, N)$ such that if a set of $N$ points in $\Omega_{3}$ satisfies $\Delta(\wp)<\Delta_{0}(t, N)$, then a spherical $t$-design $\wp^{*}$ exists near $\wp$.

The search was conducted by choosing a symmetry group from the lists of decomposable rotation groups of orders up to 21 and all indecomposable rotation groups, picking a random starting configuration invariant under this group, and optimizing with respect to the above criterion in such a way as to preserve (or increase) the symmetry. The program 
Table 1. Conjectured values of $\tau(N)$, the largest $t$ for which an $N$-point configuration on the sphere in three dimensions forms a spherical $t$-design.

\begin{tabular}{|c|c|c|c|c|c|}
\hline$N$ & $\tau(N)$ & Proof & Group & Order & Orbits (description) \\
\hline 1 & 0 & V1 & $\infty$ & $\infty$ & 1 (single point) \\
\hline 2 & 1 & V1 & $\infty$ & $\infty$ & 2 (two antipodal points) \\
\hline 3 & 1 & V1 & {$[2,3]$} & 12 & 3 (equilateral triangle) \\
\hline 4 & 2 & V1 & {$[3,3]$} & 24 & 4 (regular tetrahedron) \\
\hline 5 & 1 & V1 & {$[2,3]$} & 12 & $3+2$ (triangular bipyramid) \\
\hline 6 & 3 & V1 & {$[3,4]$} & 48 & 6 (regular octahedron) \\
\hline 7 & 2 & {$[23]$} & [3] & 6 & $3^{2}+1$ \\
\hline 8 & 3 & V1 & {$[3,4]$} & 48 & 8 (cube) \\
\hline 9 & 2 & [23] & {$[2,3]$} & 12 & $6+3$ (triangular biprism) \\
\hline 10 & 3 & [2] & {$\left[2^{+}, 10\right]$} & 20 & 10 (pentagonal prism) \\
\hline 11 & 3 & {$[2]$} & {$[2,3]^{+}$} & 6 & $6+3+2$ \\
\hline 12 & 5 & V1 & {$[3,5]$} & 120 & 12 (regular icosahedron) \\
\hline 13 & 3 & {$[2]$} & [4] & 8 & $4^{3}+1$ \\
\hline 14 & 4 & [16] & {$[2,3]^{+}$} & 6 & $6^{2}+2$ \\
\hline 15 & 3 & [2] & {$[2,5]$} & 20 & $10+5$ \\
\hline 16 & 5 & [16] & {$[3,3]^{+}$} & 12 & $12+4$ (hexakis truncated tetrahedron) \\
\hline 17 & 4 & {$[16]$} & {$[2,3]^{+}$} & 6 & $6^{2}+3+2$ \\
\hline 18 & 5 & [25] & {$\left[2^{+}, 6\right]$} & 12 & $12+6$ \\
\hline 19 & 4 & [16] & [3] & 6 & $6^{2}+3^{2}+1$ \\
\hline 20 & 5 & V1 & {$[3,5]$} & 120 & 20 (regular dodecahedron) \\
\hline 21 & 4 & {$[16]$} & {$[2,3]$} & 12 & $12+6+3$ \\
\hline 22 & 5 & [25] & {$\left[2^{+}, 10\right]$} & 20 & $10^{2}+2$ \\
\hline 23 & 5 & $\mathrm{~V} 2$ & {$[2,3]^{+}$} & 6 & $6^{3}+3+2$ \\
\hline 24 & 7 & {$[22]$} & {$[3,4]^{+}$} & 24 & 24 (improved snub cube) \\
\hline 25 & 5 & V1 & {$[2,5]^{+}$} & 10 & $10^{2}+5$ \\
\hline 26 & 6 & V3 & {$[2,3]^{+}$} & 6 & $6^{4}+2$ \\
\hline 27 & 5 & [25] & {$[2,3]$} & 12 & $12^{2}+3$ \\
\hline 28 & 6 & V3 & {$\left[2^{+}, 4\right]$} & 8 & $8^{3}+4$ \\
\hline 29 & 6 & V3 & {$[2]^{+}$} & 2 & $2^{14}+1$ \\
\hline 30 & 7 & V1 & {$[3,4]^{+}$} & 24 & $24+6$ (tetrakis snub cube) \\
\hline 31 & 6 & V3 & {$[5]^{+}$} & 5 & $5^{6}+1$ \\
\hline 32 & 7 & V1 & {$[3,4]^{+}$} & 24 & $24+8($ snub cube + cube $)$ \\
\hline 33 & 6 & V3 & {$[2,3]^{+}$} & 6 & \\
\hline 34 & 7 & V3 & {$[2,4]^{+}$} & 8 & \\
\hline 35 & 6 & V3 & {$[2,5]^{+}$} & 10 & $10^{3}+5$ \\
\hline 36 & 8 & V3 & {$[3,3]^{+}$} & 12 & $12^{3}$ (three snub tetrahedra) \\
\hline 37 & 7 & V3 & {$[3]^{+}$} & 3 & \\
\hline 38 & 7 & V3 & {$[3,4]^{+}$} & 24 & $24+8+6$ \\
\hline 39 & 7 & V3 & {$[2,3]^{+}$} & 6 & \\
\hline 40 & 8 & V3 & {$[3,3]^{+}$} & 12 & $12^{3}+4$ \\
\hline 41 & 7 & V3 & {$[2,3]^{+}$} & 6 & \\
\hline 42 & 8 & V3 & {$[2,4]^{+}$} & 8 & \\
\hline 43 & 7 & V3 & {$[6]^{+}$} & 6 & \\
\hline 44 & 8 & V3 & {$[3,3]^{+}$} & 12 & $12^{3}+4^{2}$ \\
\hline 45 & 8 & V3 & {$[2]^{+}$} & 2 & \\
\hline 46 & 8 & V3 & {$[2,4]^{+}$} & 8 & \\
\hline 47 & 8 & V3 & {$[2,3]^{+}$} & 6 & \\
\hline 48 & 9 & $\mathrm{~V} 1$ & {$[3,4]^{+}$} & 24 & $24^{2}$ (two snub cubes) \\
\hline 49 & 8 & V3 & {$[4]^{+}$} & 4 & \\
\hline 50 & 9 & V3 & {$[2,6]^{+}$} & 12 & $12^{4}+2$ \\
\hline
\end{tabular}


Table 1 (continued)

\begin{tabular}{|c|c|c|c|c|c|}
\hline$N$ & $\tau(N)$ & Proof & Group & Order & Orbits (description) \\
\hline 51 & 8 & v3 & {$[2,3]^{+}$} & 6 & \\
\hline 52 & 9 & v3 & {$[3,3]^{+}$} & 12 & $12^{4}+4$ \\
\hline 53 & 8 & v3 & {$[2,3]^{+}$} & 6 & \\
\hline 54 & 9 & V3 & {$[3,4]^{+}$} & 24 & $24^{2}+6$ \\
\hline 55 & 9 & V3 & {$[2]^{+}$} & 2 & \\
\hline 56 & 9 & v3 & {$\left[3^{+}, 4\right]$} & 24 & $24^{2}+8$ \\
\hline 57 & 9 & V3 & {$[2,3]^{+}$} & 6 & \\
\hline 58 & 9 & V3 & {$[2,4]^{+}$} & 8 & \\
\hline 59 & 9 & v3 & {$[2,3]^{+}$} & 6 & \\
\hline 60 & 10 & v3 & {$[3,3]^{+}$} & 12 & $12^{5}$ (five snub tetrahedra) \\
\hline 61 & 9 & v3 & {$[6]^{+}$} & 6 & \\
\hline 62 & 10 & v3 & {$[2,3]^{+}$} & 6 & \\
\hline 63 & 9 & V3 & {$[2.7]^{+}$} & 14 & $14^{4}+7$ \\
\hline 64 & 10 & v3 & {$[3,3]^{+}$} & 12 & $12^{5}+4$ \\
\hline 65 & 10 & V3 & {$[2]^{+}$} & 2 & \\
\hline 66 & 10 & v3 & {$[2,4]^{+}$} & 8 & \\
\hline 67 & 10 & v3 & {$[2]^{+}$} & 2 & \\
\hline 68 & 10 & V3 & {$\left[2^{+}, 4\right]$} & 8 & \\
\hline 69 & 10 & v3 & {$[4]^{+}$} & 4 & \\
\hline 70 & 11 & v3 & {$[2,5]^{+}$} & 10 & $10^{7}$ \\
\hline 71 & 10 & v3 & {$\left[2,3^{+}\right]$} & 6 & \\
\hline 72 & 11 & v3 & {$[3,5]^{+}$} & 60 & $60+12$ (pentakis truncated icosahedron) \\
\hline 73 & 10 & v3 & {$[4]^{+}$} & 4 & \\
\hline 74 & 11 & v3 & {$[2,6]^{+}$} & 12 & $12^{6}+2$ \\
\hline 75 & 11 & v3 & {$[2]^{+}$} & 2 & \\
\hline 76 & 11 & V3 & {$[3.3]^{+}$} & 12 & $12^{6}+4$ \\
\hline 77 & 11 & v3 & {$[4]^{+}$} & 4 & \\
\hline 78 & 11 & v3 & {$[3,4]^{+}$} & 24 & $24^{3}+6$ \\
\hline 79 & 11 & V3 & {$[2]^{+}$} & 2 & \\
\hline 80 & 11 & V3 & {$[3,5]^{+}$} & 60 & $60+20$ (hexakis truncated icosahedron) \\
\hline 81 & 11 & V3 & {$[4]^{+}$} & 4 & \\
\hline 82 & 11 & V3 & {$\left[2^{+}, 10^{+}\right]$} & 10 & $10^{8}+2$ \\
\hline 83 & 11 & v3 & {$[2.3]^{+}$} & 6 & \\
\hline 84 & 12 & V3 & {$[3,3]^{+}$} & 12 & $12^{7}$ (seven snub tetrahedra) \\
\hline 85 & 11 & v3 & {$[2,5]^{+}$} & 10 & \\
\hline 86 & 12 & V3 & {$[2,2]^{+}$} & 4 & \\
\hline 87 & 12 & V3 & {$[1]^{+}$} & 1 & \\
\hline 88 & 12 & v3 & {$[3,3]^{+}$} & 12 & $12^{7}+4$ \\
\hline 89 & 12 & V3 & {$[2]^{+}$} & 2 & \\
\hline 90 & 12 & V3 & {$[2,4]^{+}$} & 8 & \\
\hline 91 & 12 & v3 & {$[2]^{+}$} & 2 & \\
\hline 92 & 12 & V3 & {$[3,3]^{+}$} & 12 & $12^{7}+4^{2}$ \\
\hline 93 & 12 & v3 & {$[4]^{+}$} & 4 & \\
\hline 94 & 13 & V3 & {$\left[2^{+}, 2^{+}\right]$} & 2 & \\
\hline 95 & 12 & V3 & {$[2]^{+}$} & 2 & \\
\hline 96 & 13 & v3 & {$[3,3]^{+}$} & 12 & $12^{8}$ (eight snub tetrahedra) \\
\hline 97 & 12 & V3 & {$[4]^{+}$} & 4 & \\
\hline 98 & 13 & V3 & {$[2,4]^{+}$} & 8 & \\
\hline 99 & 12 & V3 & [2] & 4 & \\
\hline 100 & 13 & V3 & {$[3,3]^{+}$} & 12 & $12^{8}+4$ \\
\hline
\end{tabular}


cycled through the values of $N$ from 10 to 100 . For each $N$, equal effort was spent in trying to increase the value of $t$, and in trying to find a larger group for the current $t$. The search was terminated when no further improvements were found after several months of computing.

In Sections 3 and 4 we describe in more detail several of the designs mentioned in Table 1. Numerical coordinates for all these designs have been placed in the Netlib archive, and can be accessed via electronic mail, ftp, or through the World Wide Web. The designs are in the directory att/math/sloane/sphdesigns. Instructions for obtaining them can be obtained by sending the message send getting.stuff from att /math to netliberesearch. att.com.

\section{The Improved Snub Cube}

The regular snub cube [7], [9], the familiar Archimedean solid with equal edges, has symmetry group $[3,4]^{+}$. We take this group to consist of all even permutations of the three coordinates combined with any even number of sign changes, and all odd permutations combined with any odd number of sign changes. Then the vertices of the regular snub cube consist of the point $(A, B, C)$ and its images under the group, where $A=0.8503 \ldots$, $B=0.4623 \ldots, C=0.2514 \ldots$ are the unique positive roots of

$$
\begin{aligned}
& 7 Z^{6}+Z^{4}-3 Z^{2}-1 \\
& 7 Z^{6}-3 Z^{4}+5 Z^{2}-1 \\
& 7 Z^{6}-19 Z^{4}+17 Z^{2}-1
\end{aligned}
$$

respectively, and $A^{2}+B^{2}+C^{2}=1$. It may be verified from (2) that these 24 points form a spherical 3-design but not a 4-design.

However, by moving the vertices slightly, we can obtain a 7-design. Again we take the vertices to consist of the 24 images of $(A, B, C)$ under the group, where $A^{2}+B^{2}+C^{2}=1$. Equation (3b) with $\bar{s}=3$ is trivially satisfied, and (3a) with $s=3$ leads to the equations $A^{6}+B^{6}+C^{6}=\frac{3}{7}, A^{4}\left(B^{2}+C^{2}\right)+B^{4}\left(C^{2}+A^{2}\right)+C^{4}\left(A^{2}+B^{2}\right)=\frac{6}{35}, A^{2} B^{2} C^{2}=\frac{1}{105}$. It is easy to show that these equations are satisfied by taking $A=0.86624682 \ldots$, $B=0.42251865 \ldots, C=0.26663540 \ldots$ to be the positive roots of the single equation

$$
105 Z^{6}-105 Z^{4}+21 Z^{2}-1
$$

The convex hull of these points is the "improved" snub cube, differing from the regular one in that each square face has been slightly shrunk and each triangular face slightly expanded. It is almost indistinguishable in appearance from the regular snub cube. As far as we know this polyhedron is new. ${ }^{3}$

\footnotetext{
${ }^{3}$ (Added later.) The polyhedron still seems to be new. However, Bruce Reznick has pointed out to us that this spherical 7-design was first found by McLaren in 1963 [22], [26, p. 298], [24, pp. 112-113]. This is a very nice design, and we give it up grudgingly.
} 


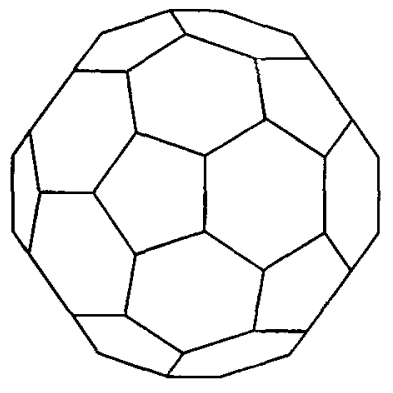

(a)

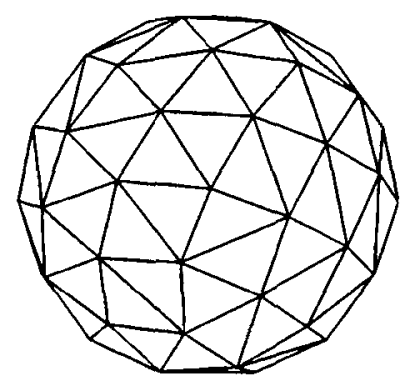

(b)

Fig. 1. (a) The regular truncated icosahedron (or soccer ball), whose 60 vertices form a spherical 5-design. The Goethals-Seidel improved football [14], which forms a spherical 9-design, is almost indistinguishable from this. (b) Our 60-point spherical 10-design.

In 1981 Goethals and Seidel [14] had shown that a similar improvement can be made to the regular truncated icosahedron (or soccer ball), another of the Archimedean solids. The 60 vertices of the Archimedean solid form a spherical 5-design, but Goethals and Seidel showed that a slight perturbation of the vertices (while preserving the group) changes them to a 9-design. Again the improved version is almost indistinguishable from the original, which is shown in Fig. 1(a). However, as can be seen from Table 1, it is possible to find a 9-design with only 48 points, and a 10-design with 60 points. The convex hull of our 60-point 10-design is shown in Fig. 1(b). Coordinates will be found in Section 6. This figure has symmetry group $[3,3]^{+}$, and is the union of five snub tetrahedra. It has 174 edges and 116 triangular faces, and we do not expect it to replace the standard soccer ball!

\section{Other Examples of New Spherical Designs}

We begin with two 5-designs that Reznick [25] was not able to find. As might be expected, these are somewhat complicated.

A 25-point 5-design with group $[2,5]^{+}$of order 10 . There are infinitely many 25-point 5 -designs, of which the following is the nicest we have found. The points are

$$
\begin{array}{rcc}
(0 & \cos k \theta & \sin k \theta), \\
\left(h_{1}\right. & -g_{1} \cos k \theta & \left.-g_{1} \sin k \theta\right), \\
\left(-h_{1}\right. & -g_{1} \cos k \theta & \left.g_{1} \sin k \theta\right), \\
\left(h_{2}\right. & g_{2} \cos \left(k \theta+\pi_{2}\right) & \left.g_{2} \sin \left(k \theta+\pi_{2}\right)\right), \\
\left(-h_{2}\right. & g_{2} \cos \left(k \theta+\pi_{2}\right) & \left.-g_{2} \sin \left(k \theta+\pi_{2}\right)\right),
\end{array}
$$

where $0 \leq k \leq 4, \theta=2 \pi / 5$,

$$
g_{1}, g_{2}=\frac{1}{2 \sqrt{3}} \sqrt{7 \mp \sqrt{11}}=0.5540 \ldots, 0.9272 \ldots,
$$


$h_{1}=\sqrt{1-g_{1}^{2}}=0.8325 \ldots, h_{2}=\sqrt{1-g_{2}^{2}}=0.3745 \ldots, \pi_{2}=2.057 \ldots$ radians, defined by the condition that $\cos \left(\pi_{2}\right)=-0.4670 \ldots$ is a root of

$$
16 Z^{5}-20 Z^{3}+5 Z-\left(\frac{30-7 \sqrt{11}}{19}\right)^{5 / 2}+\frac{1}{2}\left(\frac{6(7-\sqrt{11})}{19}\right)^{5 / 2}
$$

(It is straightforward to show that these values satisfy the equations obtained when (4) is substituted in (3a) and (3b) with $s=\bar{s}=2$.) Other solutions can be obtained by including a phase angle $\pi_{1}$ in the second and third lines of (4).

A 23-point 5-design with a group of order 2 . We must satisfy (3a), (3b) with $s=\bar{s}=$ 2. After a considerable amount of experimenting we found a numerical solution with a symmetry of order 2 , consisting of the points

$$
( \pm 1,0,0),(0, \pm 1,0),(0,0,-1),\left( \pm a_{i}, \pm b_{i}, c_{i}\right),
$$

where $0 \leq i \leq 8$, the \pm signs in the last expression are linked, $a_{0}=\frac{1}{3}$,

$$
a_{i}^{2}+b_{i}^{2}+c_{i}^{2}=1, \quad 0 \leq i \leq 8,
$$

and the approximate values of the 26 unknowns $a_{1}, \ldots, a_{8}, b_{0}, \ldots, b_{8}, c_{0}, \ldots, c_{8}$ are

$$
\begin{aligned}
& 0.5654,0.1949,0.8837,0.6521,0.5610,0.7414,0.1927,0.5854,-0.2194, \\
& 0.3485,-0.7812,-0.4754,0.7082,-0.7301,0.4805,0.7199,-0.2092,-0.9169, \\
& -0.7476,-0.5931,-0.2807,-0.2705,0.3903,0.4685,0.6668,0.7833,
\end{aligned}
$$

respectively (only four decimal places are shown, although we worked with 12 places). Substituting the symbolic forms (5) (with $a_{0}=\frac{1}{3}$ ) into (3a), (3b) with $s=\bar{s}=2$ produces 17 further equations, a typical one being

$$
\frac{1}{9} c_{0}^{2}+\sum_{i=1}^{8} a_{i}^{2} c_{i}^{2}=\frac{23}{30}
$$

We then used interval Newton methods, as implemented in the software package Intbis [20], to show that these 26 equations have a unique solution in a small box around the point (7). We later found numerical solution with a larger group, $[2,3]^{+}$, of order 6 (see Table 1), but we have included the above existence proof as illustrative of the interval method.

30- and 32-point 7-designs with group $[3,4]^{+}$. These are similar to the improved snub cube described in Section 3. For 30 points we take the $24+6$ images of the points $(A, B, C),(1,0,0)$ under the group, where $A=0.7980 \ldots, B=0.5488 \ldots, C=$ $0.2492 \ldots$ are the positive roots of $84 Z^{6}-84 Z^{4}+21 Z^{2}-1$. For 32 points we take the $24+8$ images of $(A, B, C),(1 / \sqrt{3}, 1 / \sqrt{3}, 1 / \sqrt{3})$, where $A=0.8989 \ldots, B=$ $0.4355 \ldots, C=0.0480 \ldots$ are the positive roots of $2835 Z^{6}-2835 Z^{4}+441 Z^{2}-1$. In both cases it is easy to show using Maple [5] (in particular its Gröbner basis package) that (3a) and (3b) are satisfied. 
A 48-point 9-design with group $[3,4]^{+}$. Similar to the previous examples, but now we take the images of $(A, B, C),(D, E, F)$, where $A=0.9334 \ldots, B=0.3535 \ldots$, $C=-0.0620 \ldots, D=0.7068 \ldots, E=0.6397 \ldots, F=0.3018 \ldots$ are roots of the irreducible polynomial

$$
\begin{aligned}
& 8141081016796875 Z^{36}-48846486100781250 Z^{34}+131885512472109375 Z^{32} \\
& \quad-212133311066250000 Z^{30}+226833777359437500 Z^{28} \\
& -170368273215000000 Z^{26}+92508869648475000 Z^{24} \\
& -36735117403950000 Z^{22}+10602550092251250 Z^{20}-2145915231232500 Z^{18} \\
& +270106833039750 Z^{16}-9766335726000 Z^{14}-3473862884100 Z^{12} \\
& +770657554800 Z^{10}-80424958320 Z^{8}+4880358000 Z^{6} \\
& -168429429 Z^{4}+2733318 Z^{2}-8269 .
\end{aligned}
$$

The complexity of this polynomial indicates why we have been satisfied to find purely numerical solutions for the larger designs in the table.

\section{Designs with Larger Numbers of Points}

Although Table 1 only extends to $N=100$, larger designs for fixed $t$ may be obtained using the fact that an $N_{1}$-point design and an $N_{2}$-point design can be combined to form an $\left(N_{1}+N_{2}\right)$-point design. For example $N$-point 6-designs can be found for all $N \geq 28$ by combining the designs in the table.

Alternative (and exact) designs can be found using a construction of Bajnok [1]. An $n$-point interval $t$-design consists of $n$ distinct points $P_{1}, \ldots, P_{n}$ with $-1 \leq P_{i} \leq 1$ such that

$$
\frac{1}{2} \int_{-1}^{1} f(x) d x=\frac{1}{n} \sum_{i=1}^{n} f\left(P_{i}\right)
$$

holds for all polynomials $f$ of degree $\leq t$. Bajnok shows that by taking regular $m$-gons at latitudes $P_{1}, \ldots, P_{n}$ a three-dimensional $m n$-point spherical $t$-design is obtained, provided $m \geq t+1$.

It is known (see the survey by Gautschi [11]) that, for $t=1,2,3, \ldots, 11, n$-point interval $t$-designs exist for all $n \geq 1,2,2,4,4,6,6,9,9,13,13$, respectively. When $t=6$, for example, Bajnok's construction produces $N$-point spherical 6-designs with $N=42,48,49,54, \ldots$ and all $N \geq 108$.

\section{A Conjecturally Infinite Family of $t$-Designs}

Inspection of Table 1 shows that there is a sequence of $N=12 \mathrm{~m}$-point spherical $t$ designs with group $G=[3,3]^{+}$(or larger) which for $m=2, \ldots, 8$ have $t=7, \ldots, 13$. It might naively be expected that this sequence continue in a linear fashion, but the true situation is more complicated.

A full orbit under $G$ can be taken to consist of 12 points $( \pm A, \pm B, \pm C),( \pm B, \pm C$, $\pm A),( \pm C, \pm A, \pm B)$, where the product of the signs is positive and $A^{2}+B^{2}+C^{2}=1$. 
(Their convex hull is a snub tetrahedron.) So a set $\wp$ which is the union of $m$ full orbits under $G$ contains $2 m$ degrees of freedom.

Consider the ring $R$ of polynomials in $X, Y, Z$ that are invariant under $G$, ignoring the trivial invariant $X^{2}+Y^{2}+Z^{2}$. If $R_{j}$ is the subspace of $R$ consisting of homogeneous invariants of degree $j$, then the dimensions $d_{j}=\operatorname{dim} R_{j}$ are given by the Molien series for $G$ :

$$
\Phi(\lambda)=\frac{1+\lambda^{6}}{\left(1-\lambda^{3}\right)\left(1-\lambda^{4}\right)}=\sum_{j=0}^{\infty} d_{j} \lambda^{j}
$$

(see Table 10 of [8]).

In order for $\wp$ to form a $t$-design it is necessary and sufficient that the average of $f$ over $\wp$ is equal to the average of $f$ over $\Omega_{3}$ for all $f \in R_{1} \cup R_{2} \cup \cdots \cup R_{t}$ [12], [14]. This imposes

$$
e_{t}=d_{1}+d_{2}+\cdots+d_{t}
$$

conditions on $\wp$. So provided $2 m \geq e_{1}$, we may reasonably expect that it will be possible to choose the orbits so that all the conditions are satisfied, and then a $t$-design with $N=12 m$ points will exist. The values of $e_{t}$ can be obtained by expanding (8), and we discover that $t$-designs with $N=12 \mathrm{~m}$ points should exist for the values of $t$ and $N$ shown in Table 2. Table 1 shows that such designs do indeed exist for $t \leq 13$ (in fact for $t=7$ only 24 points are needed). We have verified numerically that the predicted designs also exist for all $t \leq 21$, and Table 3 gives a set of orbit representatives $(A, B, C)$ for a selection of these designs. (The others can be obtained through Netlib-see Section 2.)

An explicit formula for $e_{t}$ (for $t \geq 6$ ) can be found from (8) and (9):

$$
\begin{aligned}
e_{t}= & {\left[\frac{t}{12}\right]\left(t-6\left[\frac{t}{12}\right]-5\right)+\left[\frac{t-6}{12}\right]\left(t-6-6\left[\frac{t-6}{12}\right]-5\right) } \\
& +9\left[\frac{t}{12}\right]+9\left[\frac{t-6}{12}\right]+A_{t \bmod 12}+A_{(t-6) \bmod 12}+1
\end{aligned}
$$

where $A_{0}, \ldots, A_{11}$ are $0,0,0,1,2,2,3,4,5,6,7,8$. Therefore, if these designs continue to exist, we will have a sequence of $t$-designs with $N=12 \mathrm{~m}$ points satisfying $N=\left(t^{2} / 2\right)(1+o(1))$ as $t \rightarrow \infty$. Incidentally, Korevaar and Meyers [21] show that $t$ designs with $N=O\left(t^{3}\right)$ points exist and conjecture that $N=O\left(t^{2}\right)$ should be possible. Equation (2) gives a lower bound of $\left(t^{2} / 4\right)(1+o(1))$.

Table 2. Beginning of conjecturally infinite sequence of three-dimensional spherical $t$-designs with $N=12 \mathrm{~m}$ points and group $[3,3]^{+}$. These have been constructed numerically for $t \leq 21$ (see Table 3).

\begin{tabular}{rrrrrrrrrrrr}
\hline$t$ & 3 & 4 & 5 & 6 & 7 & 8 & 9 & 10 & 11 & 12 & 13 \\
$N$ & 12 & 12 & 12 & 24 & 36 & 36 & 48 & 60 & 72 & 84 & 96 \\
& & & & & & & & & & & \\
$t$ & 14 & 15 & 16 & 17 & 18 & 19 & 20 & 21 & 22 & 23 & 24 \\
$N$ & 108 & 132 & 144 & 156 & 180 & 204 & 216 & 240 & 264 & 288 & 312 \\
\hline
\end{tabular}


Table 3. $N=12 m$-point spherical $t$-designs formed from the union of $m$ full orbits under group $[3,3]^{+}$. The entries give a list of $m$ orbit representatives $A, B, C$.

\begin{tabular}{|c|c|c|}
\hline \multicolumn{3}{|c|}{$N=36, \quad t=8$} \\
\hline 0.74051521 & 0.24352778 & 0.62636367 \\
\hline 0.80542549 & 0.30620001 & -0.50747545 \\
\hline 0.95712033 & 0.28624872 & 0.04452356 \\
\hline \multicolumn{3}{|c|}{$N=60, \quad t=10$} \\
\hline 0.71315107 & 0.03408955 & 0.70018102 \\
\hline 0.75382867 & 0.54595191 & -0.36562119 \\
\hline 0.78335594 & -0.42686412 & -0.45181910 \\
\hline 0.93321004 & 0.12033145 & -0.33858436 \\
\hline 0.95799794 & 0.27623022 & 0.07705072 \\
\hline \multicolumn{3}{|c|}{$N=72, \quad t=11$} \\
\hline 0.66932119 & -0.65648669 & -0.34789994 \\
\hline 0.75683290 & 0.38164750 & -0.53061205 \\
\hline 0.82190371 & 0.54929373 & -0.15083333 \\
\hline 0.85544705 & 0.04115447 & 0.51625251 \\
\hline 0.90728126 & 0.36233033 & 0.21344190 \\
\hline 0.97885492 & 0.12557302 & -0.16147588 \\
\hline \multicolumn{3}{|c|}{$N=96, \quad t=13$} \\
\hline 0.69989534 & 0.59974524 & -0.38788163 \\
\hline 0.73338128 & -0.54971991 & -0.39994990 \\
\hline 0.78556905 & 0.09585688 & -0.61130412 \\
\hline 0.82321276 & 0.56450535 & 0.06045217 \\
\hline 0.83255539 & -0.25643858 & -0.49100996 \\
\hline 0.88122889 & 0.33818291 & -0.33025441 \\
\hline 0.96391874 & -0.26382492 & -0.03545521 \\
\hline 0.96783463 & -0.01683358 & -0.25102343 \\
\hline \multicolumn{3}{|c|}{$N=108, \quad t=14$} \\
\hline 0.69160471 & -0.40217576 & 0.59994798 \\
\hline 0.71050575 & 0.58202818 & 0.39550573 \\
\hline 0.75403890 & 0.65127837 & -0.08521631 \\
\hline 0.80598041 & 0.26283378 & 0.53039041 \\
\hline 0.86226532 & -0.39729017 & 0.31410038 \\
\hline 0.86442500 & -0.05628604 & 0.49960114 \\
\hline 0.87315060 & -0.46879380 & -0.13356797 \\
\hline 0.96418944 & 0.16093133 & 0.21080756 \\
\hline 0.97567128 & -0.17376307 & 0.13368600 \\
\hline \multicolumn{3}{|c|}{$N=144, \quad t=16$} \\
\hline 0.65758346 & 0.61920220 & 0.42915339 \\
\hline 0.70203400 & -0.68122298 & 0.20756570 \\
\hline 0.70428352 & -0.55221381 & 0.44614418 \\
\hline 0.71018481 & -0.16518988 & -0.68436090 \\
\hline 0.84130836 & -0.32306467 & 0.43339297 \\
\hline 0.84532735 & -0.30622774 & -0.43777418 \\
\hline 0.85087242 & 0.52354706 & -0.04375603 \\
\hline 0.85473787 & -0.02894596 & 0.51825216 \\
\hline 0.87135881 & 0.43350173 & 0.22980441 \\
\hline 0.94028712 & -0.28839660 & 0.18079695 \\
\hline 0.96296114 & 0.02735042 & 0.26824950 \\
\hline 0.98473889 & 0.16325742 & 0.06030199 \\
\hline
\end{tabular}


Table 3 (continued)

\begin{tabular}{lrr}
\hline & $N=240, \quad t=21$ \\
0.66536339 & 0.58086027 & 0.46892741 \\
0.67683321 & -0.48257247 & 0.55589623 \\
0.71800639 & 0.65744688 & -0.22853979 \\
0.72687147 & -0.02748828 & -0.68622319 \\
0.73733200 & -0.62085150 & -0.26624225 \\
0.77263286 & 0.51705945 & -0.36835851 \\
0.77909960 & -0.23760971 & -0.58012537 \\
0.78443181 & 0.28431902 & -0.55120724 \\
0.78559925 & -0.40515695 & -0.46763412 \\
0.81763902 & -0.57522572 & 0.02412057 \\
0.84781923 & 0.06632578 & -0.52612113 \\
0.86317647 & -0.46818182 & -0.18902953 \\
0.89265354 & -0.41253405 & 0.18161861 \\
0.89457952 & -0.27876240 & 0.34931219 \\
0.90354264 & 0.09900269 & 0.41690427 \\
0.90950707 & 0.29209374 & 0.29576703 \\
0.94298382 & 0.33269411 & 0.00980574 \\
0.95866803 & -0.10111361 & 0.26595424 \\
0.97946878 & 0.11341985 & 0.16666388 \\
0.99028895 & 0.12883316 & -0.05224764 \\
\hline
\end{tabular}

\section{Acknowledgment}

We thank Bruce Reznick for some very helpful comments on an earlier version of this paper.

\section{References}

1. B. Bajnok, Construction of designs on the 2-sphere, European J. Combin., 12 (1991), 377-382.

2. B. Bajnok, Paper presented at American Mathematical Society meeting, College Station, TX, Oct. 22, 1993 (see Abstracts Amer. Math. Soc., 14 (1993), \#886-05-35).

3. E. Bannai and R. M. Damerell, Tight spherical designs, I, J. Math. Soc. Japan, 31 (1979), 199-207.

4. E. Bannai and R. M. Damerell, Tight spherical designs, II, J. London Math. Soc., 21 (1980), 13-30.

5. B. W. Char, K. O. Geddes, G. H. Gonnet, B. L. Leong, M. B. Monagan, and S. M. Watt, Maple V Reference Manual, Springer-Verlag, New York, 1991.

6. J. H. Conway and N. J. A. Sloane, Sphere Packing, Lattices and Groups, 2nd edn., Springer-Verlag, New York, 1993.

7. H. S. M. Coxeter, Regular Polytopes, 3rd edn., Dover, New York, 1973.

8. H. S. M. Coxeter and W. O. J. Moser, Generators and Relations for Discrete Groups, 4th edn., SpringerVerlag, New York, 1984.

9. H. M. Cundy and A. P. Rollett, Mathematical Models, 2nd edn., Oxford University Press, Oxford, 1961.

10. P. Delsarte, J. -M. Goethals, and J. J. Seidel, Spherical codes and designs, Geom. Dedicata, 6 (1977), 363-388.

11. W. Gautschi, Advances in Chebyshev quadrature, in Numerical Analysis (Proc. 6th Biennial Dundee Conf., Univ. Dundee, Dundee, 1975), Lecture Notes in Mathematics, Vol. 506, Springer-Verlag, Berlin, 1976, pp. $100-121$.

12. J. -M. Goethals and J. J. Seidel, Spherical designs, in D. K. Ray-Chaudhuri, ed., Relations Between Combinatorics and Other Parts of Mathematics, Proceedings of Symposia in Pure Mathematics, Vol. 34, American Mathematical Society, Providence, RI, 1979, pp. 255-272. 
13. J. -M. Goethals and J. J. Seidel, Cubature formulae, polytopes and spherical designs, in C. Davis et al., eds., The Geometric Vein: The Coxeter Festschrifi, Springer-Verlag, New York, 1981, pp. 203-218.

14. J. -M. Goethals and J. J. Seidel, The football, Nieuw Arch. Wisk., 29 (1981), 50-58.

15. R. H. Hardin and N. J. A. Sloane, Operating Manual for Gosset: A General-Purpose Program for Constructing Experimental Designs, 2nd edn., Statistics Research Report No. 106, Bell Laboratories, Murray Hill, NJ, Oct. 1992.

16. R. H. Hardin and N. J. A. Sloane, New spherical 4-designs, Discrete Math., 106/107 (1992), 255-264.

17. R. H. Hardin and N. J. A. Sloane, A new approach to the construction of optimal designs, J. Statist. Plann. Inference, 37 (1993), 339-369.

18. R. H. Hardin, N. J. A. Sloane, and W. D. Smith, Spherical Codes, in preparation.

19. R. Hooke and T. A. Jeeves, "Direct search" solution of numerical and statistical problems, J. Assoc. Comput. Mach., 8 (1961), 212-229.

20. R. B. Kearfott and M. Novoa III, Algorithm 681: INTBIS, a portable interval Newton/bisection package, ACM Trans. Math. Software, 16 (1990), 152-157.

21. J. Korevaar and J. L. H. Meyers, Spherical Faraday cage for the case of equal point changes and Chebyshevtype quadrature, J. Integral Transforms Special Funct., 1 (1993), 105-127.

22. A. D. McLaren, Optimal numerical integration on a sphere, Math. Comp., 17 (1963), 361-383.

23. Y. Mimura, A construction of spherical 2-designs, Graphs Combin., 6 (1990), 369-372.

24. B. Reznick, Sums of Even Powers of Real Linear Forms, Memoirs of the American Mathematical Society, No. 463, American Mathematical Society, Providence, RI, 1992.

25. B. Reznick, Some constructions of spherical 5-designs, Linear Algebra Appl., 1995, to appear.

26. A. H. Stroud, Approximate Calculation of Multiple Integrals, Prentice-Hall, Englewood Cliffs, NJ, 1971.

Received December 7, 1994, and in revised form September 12, 1995. 